\title{
THE NEXT DECADE IN OBSERVATIONAL SOLAR RESEARCH
}

\author{
J. M. BECKERS
}

Sacramento Peak Observatory, AFCRL, Sunspot, N.M. 88349, U.S.A.

\section{Introduction}

The last two decades have been exceptionally fruitful for astrophysics. Because of very rapid developments, especially in the area of observational astrophysics, our view of the Universe has been substantially completed, challenged and altered. Solar Physics has very much been part of this progress. Developments in space and groundbased technology have virtually completely opened up the entire spectrum of solar electromagnetic and corpuscular radiations. Electromagnetic radiation of the Sun can now be studied from gamma ray wavelengths shorter than $10^{-4} \AA$ to radio wavelengths as long as $10^{14} \AA$, a range of approximately 60 octaves. Continuing improvements of spatial resolution of the Sun at all these wavelengths and concommitant development of our theoretical understanding of both the radiative and magnetohydrodynamic processes on the Sun have made solar physics an exciting field to those interested in the Sun per se, as well as to those who view the Sun as a star whose study aids us in the understanding of astrophysics in a broader sense.

A committee of The National Academy of Sciences in the United States under the chairmanship of J. L. Greenstein conducted between 1969 and 1971 a study of the needs of astronomy in the next decade. I took part in this study and it is probably because of this that I was asked to give this review on the next decade in observational solar physics. This review is therefore partly based on the results of the discussions in that committee and in its solar panel. Most of this review will reflect, however, my own opinions.

The first part of the Greenstein Committee's Solar Panel study was devoted to the goals of solar research and to the relevance of solar physics to other areas of human endeavor. Specifically, it outlined the significance of solar physics for general astronomy, for pure physics, for the understanding of the solar system and for human activities in the Earth's environment. It is outside the scope of this review to discuss these items in detail. It was felt, however, that it was necessary to put solar research in a broader perspective before discussing the solar physics program. Solar physics is unique among the astrophysical sciences in that in addition to being an area of pure scientific endeavor, it is of practical interest to the inhabitants of this planet. Short term solar variations associated, for example with solar flares, are the dominant factor in determining the weather in the upper atmosphere and nearby space. Longer term variations of the Sun are now considered to be related to weather changes and are a very likely cause for the ice ages.

The remainder of the Greenstein Committee Solar Panel study was devoted to the discussion of a solar physics program for the nineteen seventies. This discussion was 
so-called problem oriented. It is a general trend in discussing future programs in solar physics to outline the main problems and to base on this the requirements for future instrumentation. This way of approaching the future of a science is certainly partly valid. In looking back, however, one notices that many of the discoveries and advances in solar physics, and in astrophysics in general, were the results of technological progress and of exploration. The discovery of quasars and pulsars was not the result of a problem-oriented approach but of the desire to explore and to develop novel and new techniques. So was the discovery of many of the phenomena on the Sun, which form the basis of much of solar physics, the results of the technological and explorative approach, although one can rightly point at some solar phenomena, like the solar wind, whose discovery was related to a problem orientation.

In the Greenstein study we defined four broad major problem areas in solar physics. These were:

(a) Flare Instabilities and Particle Acceleration,

(b) Energy Generating Processes in the Solar Interior,

(c) Energy and Mass Transport in the Sun, and

(d) The Large Scale Circulation in the Sun.

All these problem areas are, at least in part, related to the topic of this review which concentrates on the Sun viewed as a star with an emphasis on the observable aspects of the quiet Sun. In this review, however, I will not strictly follow such a problemoriented approach. Instead I will concentrate on what I will call the needs of observational solar research like, for example, the need for improved spatial resolution. I will then argue for the support of these needs on the basis of problems which are to be solved.

\section{Present Status of Observational Solar Research}

Before discussing the future objectives of observational solar physics I would like to survey the present status of the field. This will help to outline the limitations of solar observations and it will indicate what the needs are for future improvements at least as far as the purely technological aspects are concerned.

The quality of solar observations can pretty much be described by their spatial, spectral and temporal resolution of solar phenomena. To achieve the highest possible resolution in one of these three dimensions one often has to sacrifice resolution in one or both of the other two. For example, the highest possible spatial resolution observation of photosphere and sunspot fine structures are only possible by very short exposures so that the high spectral resolution becomes impossible. In the ultraviolet region of the spectrum, flux limitations impose a similar limitation.

Figures 1 and 2 summarize the spatial and spectral resolution which can be (and has been) achieved with today's instrumentation. Figure 1 illustrates the spatial resolution on the Sun in arc seconds for the full 18 decade range of the observed electromagnetic spectrum. The dashed lines give the limiting resolution of telescopes of the given aperture. The best resolution on the sun is presently achieved in the optical, and to some extent, in the $\mathrm{cm}$ region of the spectrum. In the optical region a spatial resolution 


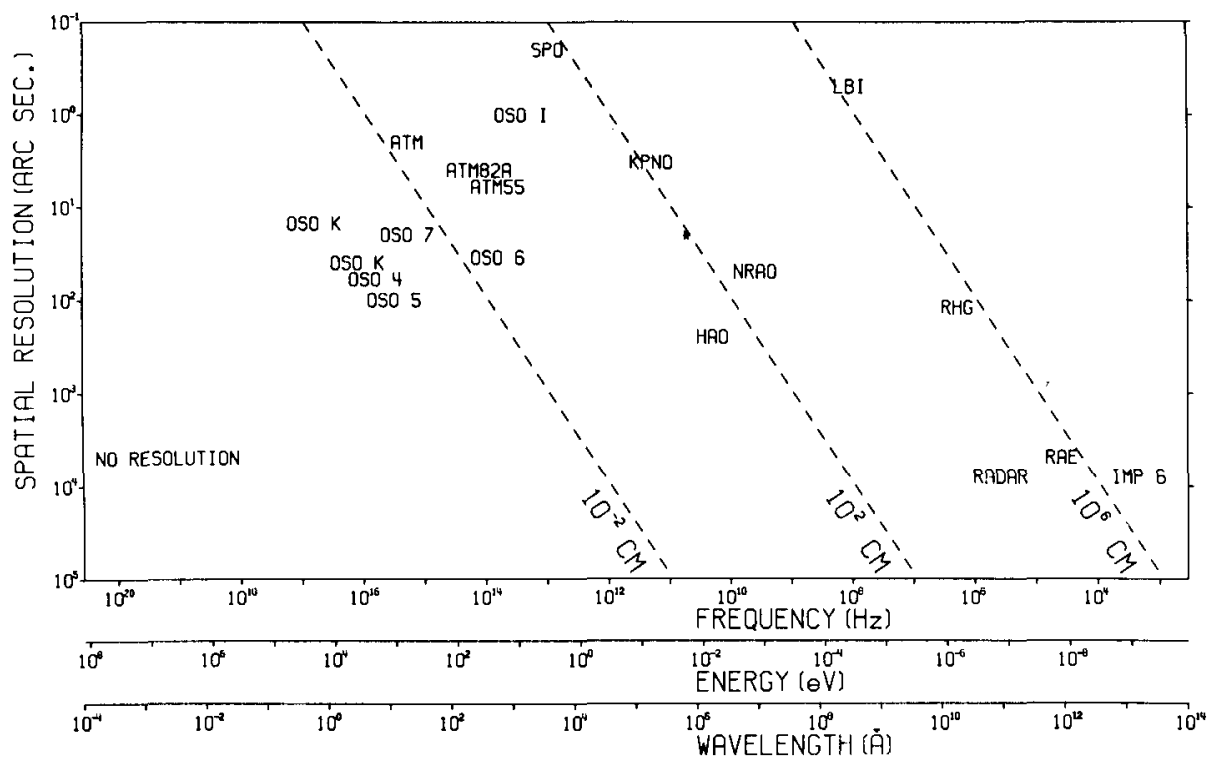

Fig. 1. Best spatial resolution achieved at different wavelengths in the solar spectrum. Dashed lines represent the limiting resolution of telescopes with the indicated aperture. Explanation of symbols: OSO $=$ Orbiting Solar Observatory: ATM $=$ Apollo Telescope Mount; SPO $=$ Sacramento Peak Observatory; KPNO $=$ Kitt Peak National Observatory; $\mathrm{HAO}=$ High Altitude Observatory; $\mathrm{NRAO}=$ National Radio Astronomy Observatory; RAE $=$ Radio Astronomy Explorer; $\mathrm{LBI}=$ Kilometer Baseline Interferometer; RHG $=$ Culgoora Radioheliograph. OSO-I and J points represent the predicted performance. OSO-K points refer to a feasibility study for a solar-flare satellite.

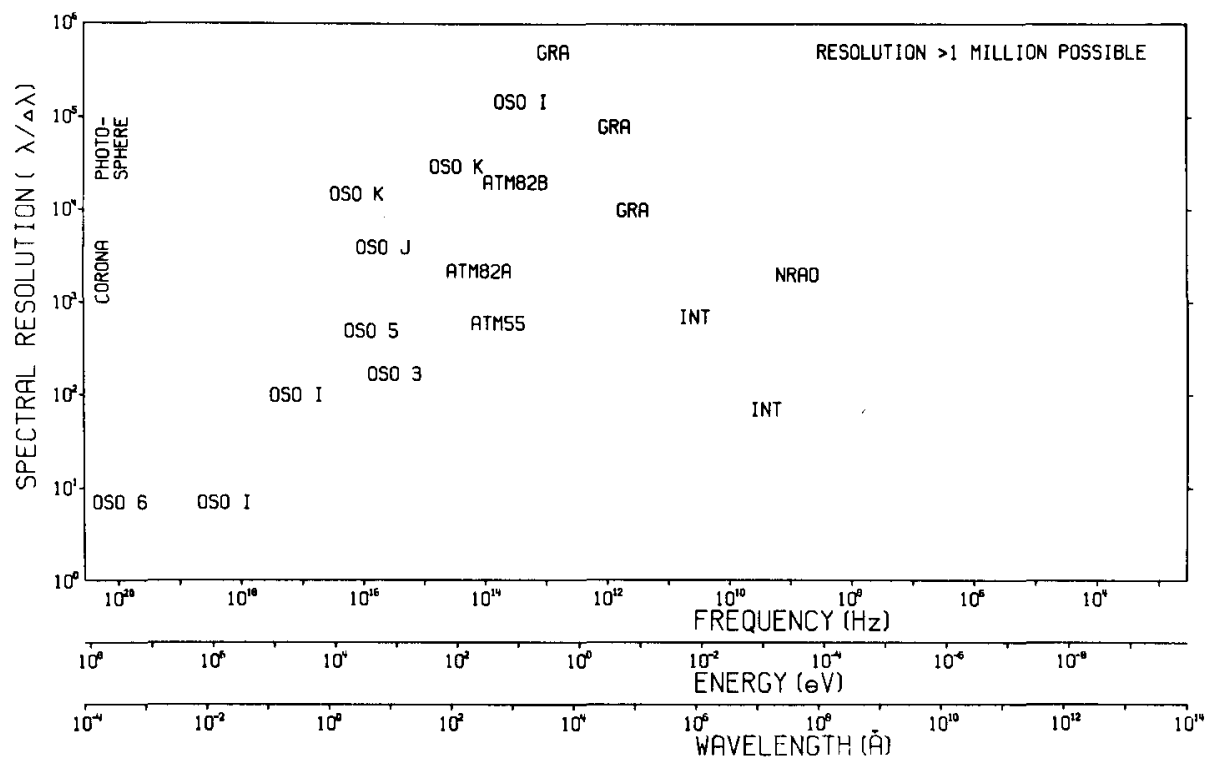

Fig. 2. Best spectral resolution achieved at different wavelengths in the solar spectrum. Explanation of symbols as in Figure 1, except for GRA = Grating Spectrograph; INT = Michelson Interferometer (Fourier spectrometer). 
of $0.2^{\prime \prime}$ corresponding to $150 \mathrm{~km}$ on the Sun can be achieved. The $\mathrm{cm}$ radio observations of 0.3 " are obtained by kilometer baseline interferometry (Kundu, 1973). Their resolution is high only in one spatial dimension. The same is true for occasional eclipse observations, not shown in this slide, which attained high spatial resolution in the ultraviolet, infrared, and $\mathrm{mm}$ region of the solar limb spectrum (see e.g. Gabriel et al., 1971; Noyes et al., 1968; Coates et al., 1958) by means of the occultation by the Moon. Only in the optical region and $\mathrm{cm}$ region of the solar spectrum is the resolution good enough to see solar details like solar granules and spicules. In the soft X-ray and EUV regionwe can resolve the chromospheric network and its coarse elements. Single radio dishes and the Culgoora radioheliograph fall just short of resolving the supergranulation and network. In the hard X-ray, and low frequency radio spectrum the resolution becomes so low that only integrated solar radiation can be studied. The same is true for the radar reflections from the Sun. I should point out again that the highest spatial resolution generally corresponds to low spectral resolution. The $0.2^{\prime \prime}$ optical resolution, for example, can only be achieved for very short exposure times as is the case for integrated light observations. Very high spectral resolution solar spectra achieve at best $0.5^{\prime \prime}$ spatial resolution.

Figure 2 summarizes the status of spectral resolution that can be obtained. Spectral resolution sufficient to resolve photospheric, chromospheric and coronal line profiles can be achieved over most of the electromagnetic spectrum. Only in the far infrared,

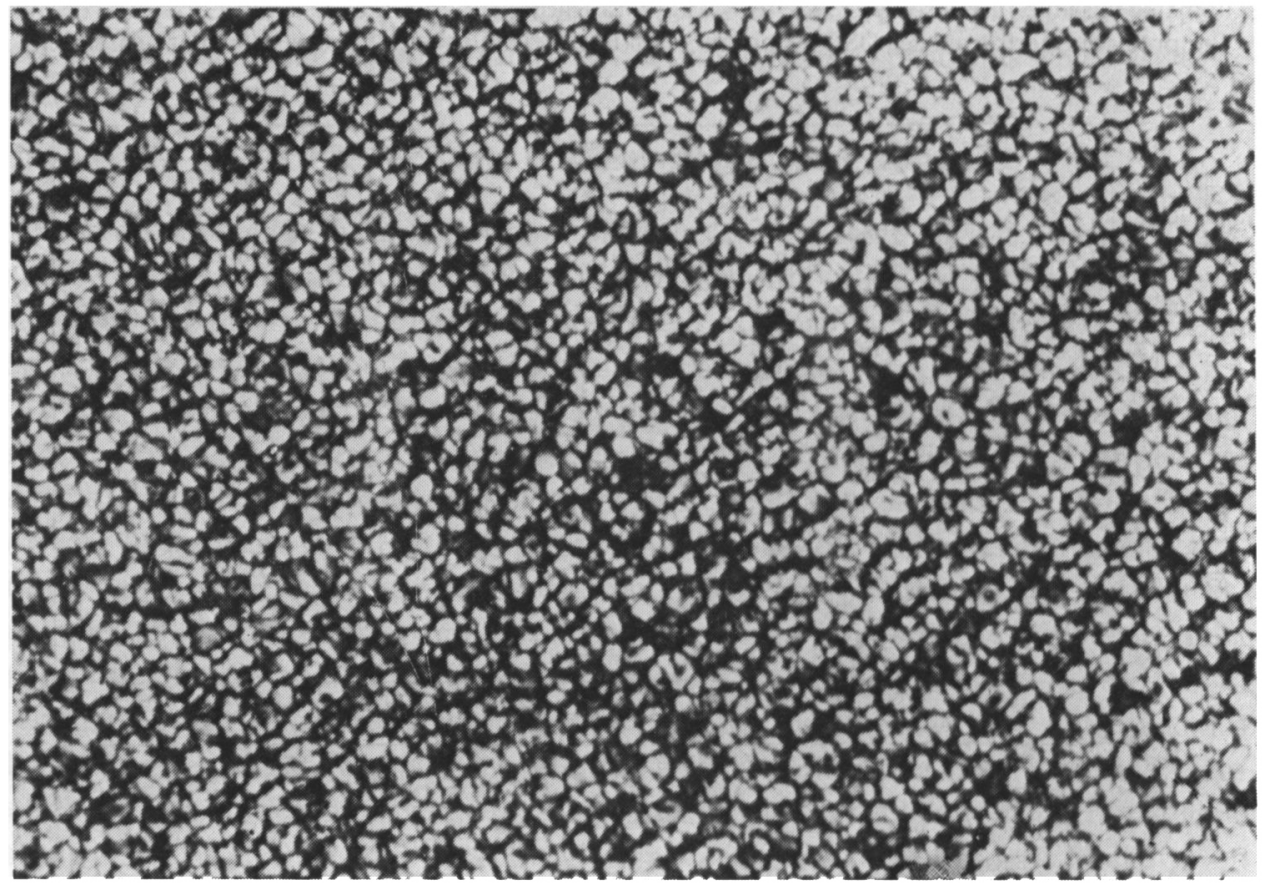

Fig. 3. Observation of the solar photosphere with the Pulkovo balloonborne telescope. (Courtesy: Pulkovo Observatory.) 
millimeter and X-ray spectrum is the spectral resolution insufficient to study the shape of solar spectral lines.

I did not prepare a figure to demonstrate the temporal resolution as a function of wavelength. Most solar variations seem to occur on time scales longer than $1 \mathrm{~s}$ so that temporal resolution is in principle no limitation. Flux limitations set an effective limit, however, for some experiments if an acceptable signal-to-noise ratio is to be obtained. The only exceptions to this are coronal variations associated with solar activity. In both the hard X-ray fluxes and in the radio emission fast unresolved coronal bursts are observed.

In the last part of this quick review of the present status of observational solar research I want to present a sample of some of the most excellent and up-to-date observations of the solar atmosphere. I will do this successively for photospheric, chromospheric, and coronal observations.

The photosphere is best observed in the optical and near infrared region of the spectrum. Only in these spectral regions is the absorption in the solar atmosphere small enough to make photospheric observations feasible. The best observations so far have been obtained from balloons and from carefully designed ground-based telescopes.

\section{A BANDWIDTH}

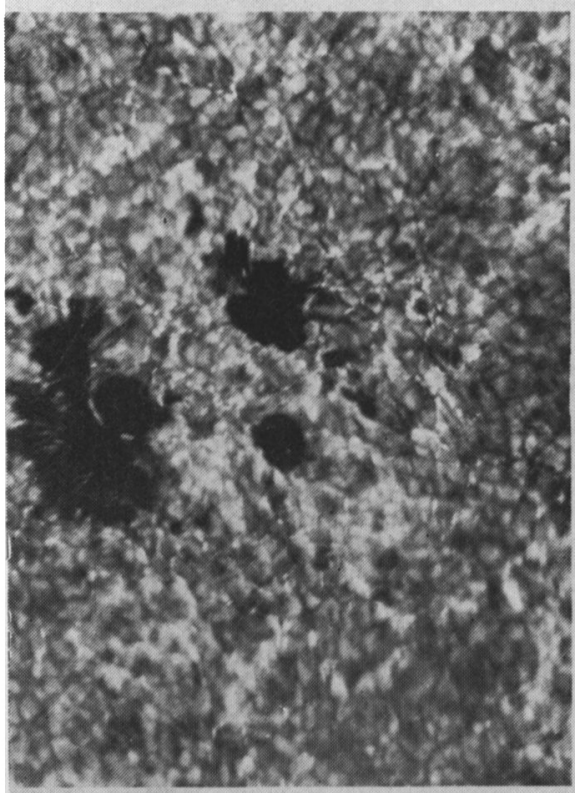

\section{A BANDWIDTH}

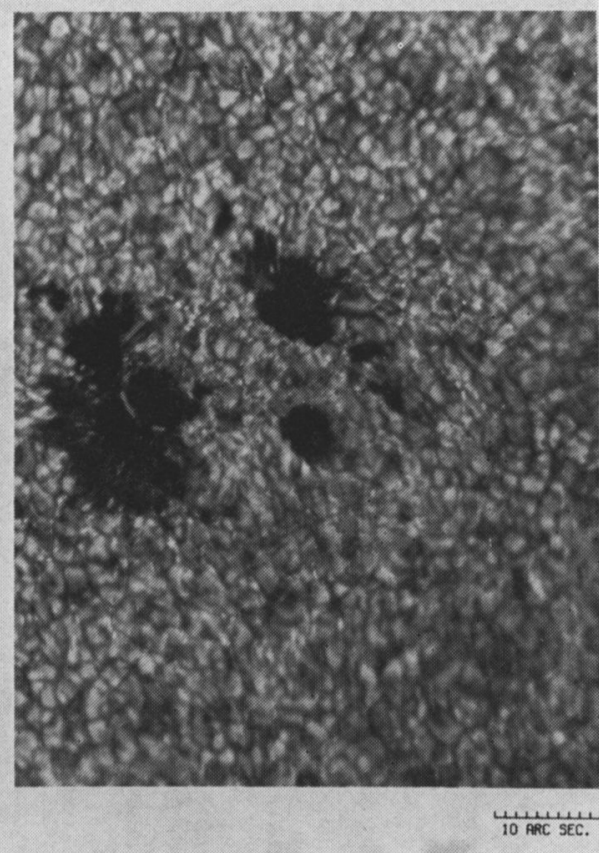

Fig. 4. Observations of the upper solar photosphere near the calcium $\mathrm{K}$ line. (Courtesy: Sacramento Peak Observatory, AFCRL.) 

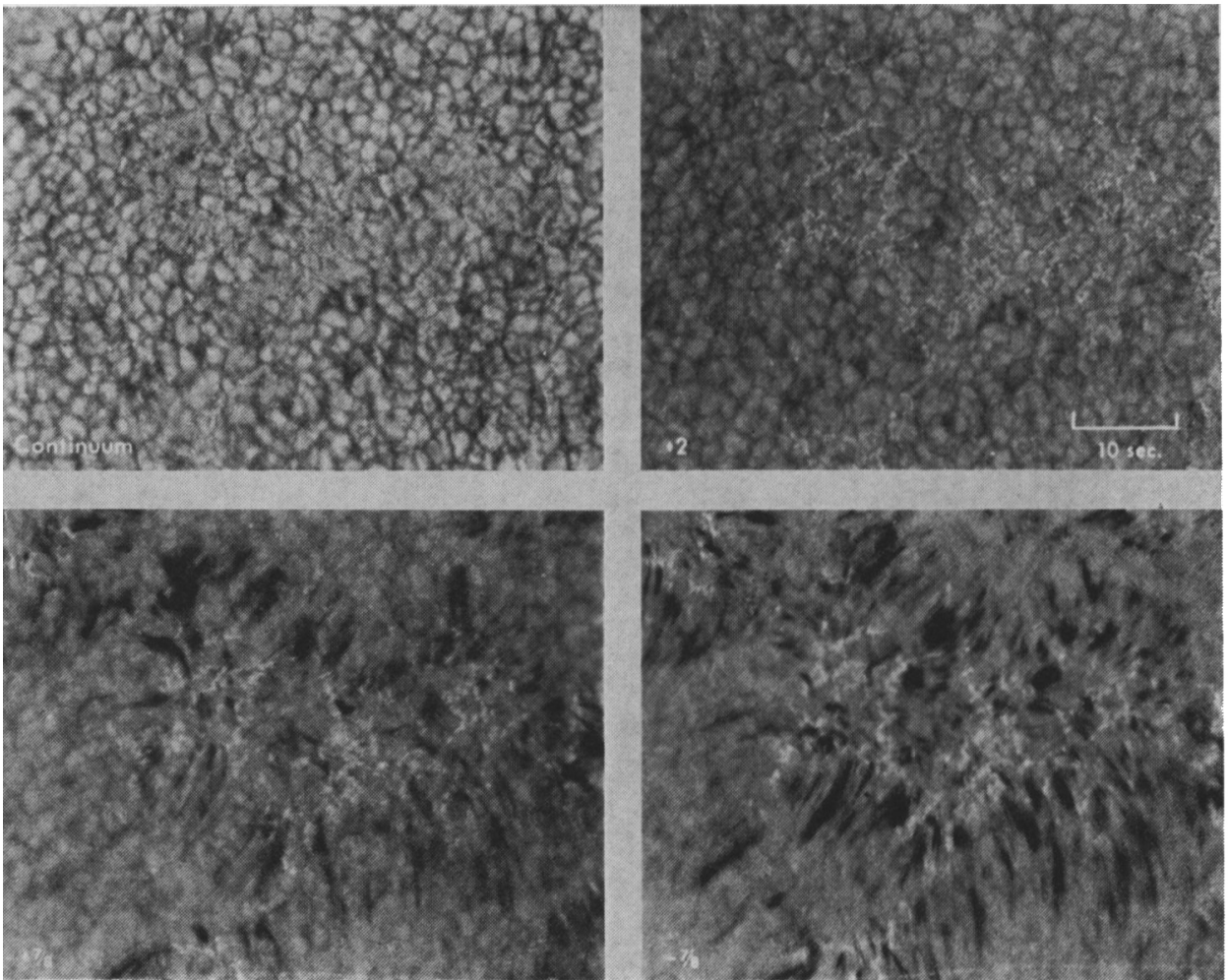

Fig. 5. Observations of solar 'filigree'. (Courtesy: Sacramento Peak Observatory, AFCRL.)

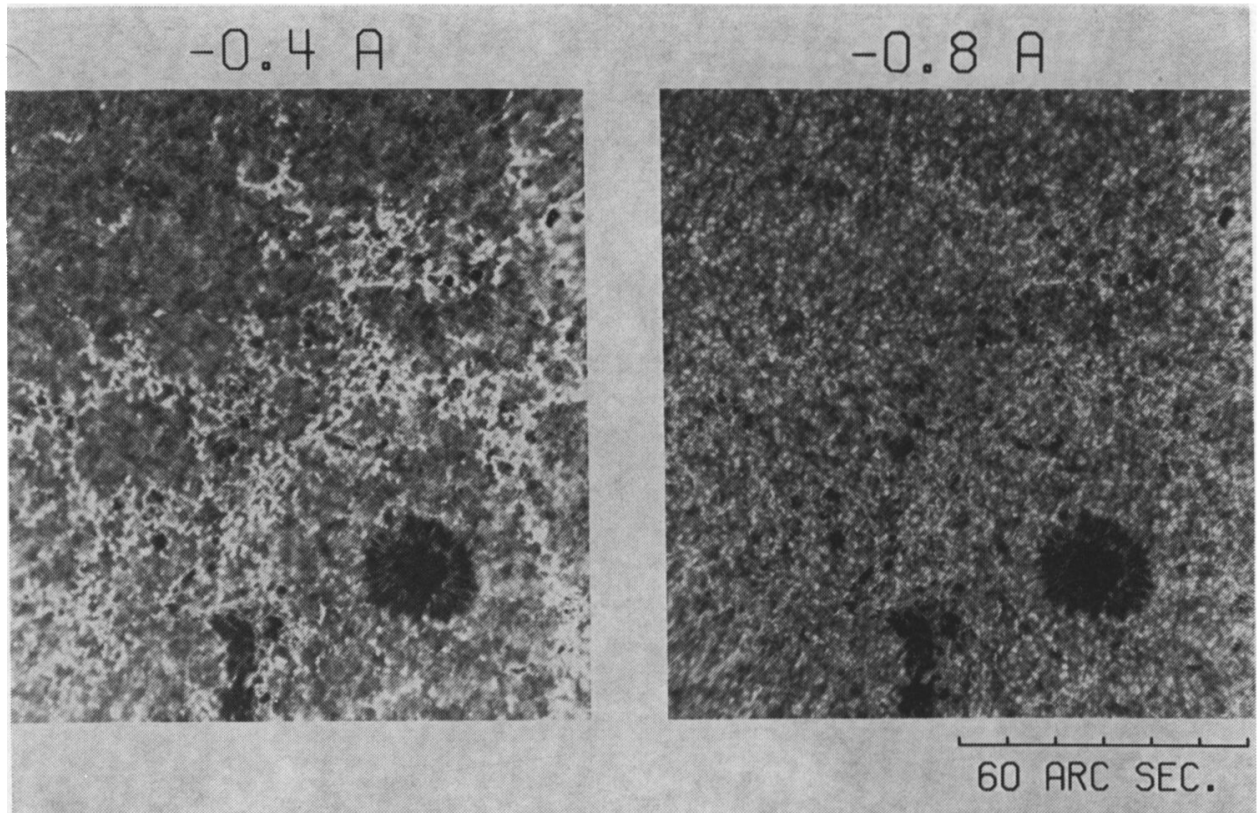

Fig. 6. Observations of subgranular structures with the Universal Birefringent Filter. (Courtesy; Sacramento Peak Observatory, AFCRL.) 
Figure 3 was taken with the balloon-borne telescope of the Pulkovo Observatory in the U.S.S.R. This telescope is equipped with a variety of auxiliary devices including a spectrograph with slit jaw monitor and an integrated light camera. This slide was made with that camera. At first sight, it appears similar in quality to those made with the Princeton stratoscope balloon project in the early 1960's. However, when I inspected the films in Leningrad, I noticed the presence of subgranular structures located mainly in the dark intergranular lanes. Because of the excellent performance of the Sacramento Peak Vacuum Telescope, it has been possible to confirm the presence of these structures. Figure 4 shows the best image of the photosphere obtained in integrated light with the vacuum telescope of Dr Mehltretter. It clearly shows the subgranular structures whose size has been measured to be $0.2^{\prime \prime}$ or less. Similar structures can be seen in Figure 5 taken by Dr Dunn in the wings of the $\mathrm{H} \alpha$ line, and in many other lines studied with the Universal Birefringent Filter (Beckers, 1972a, b) at the Observatory. Their group appearance has been called by Dr Dunn 'filigree'. Figure 6 shows an image obtained through this fully tunable Lyot filter in the wing of the Magnesium $b_{1}$ line. Similar Zeeman observations taken with the Universal Filter in the core of the magnetically very sensitive $b_{2}$ line show that the location of the filigree-subgranular structure pattern tends to coincide with regions of magnetic field enhancements. This has led to the picture of these subgranular structures as the result of the bundling of the magnetic field lines at the granule boundaries by the outward flows in the granules. This bundling occurs presumably in very much the same way as the formation of the magnetic network formed by the supergranulation. A spectroscopic investigation by Drs Simon and Zirker (1973) has as yet failed to support this association of the subgranular structure with the magnetic enhancements. Spectra require, however, longer exposure times and therefore have a lower spatial resolution which may explain this failure. The best spectra have a spatial resolution of about $0.5^{\prime \prime}$. Figure 7 shows an example of a spectrum in the chromospheric calcium $\mathrm{H}$-line. The strong enhancements in the $\mathrm{K}_{2}$ reversals (A and $\mathrm{B}$ ) are the intersection of the calcium chromospheric network. Very well visible are also the bright emission grains in the violet $\mathrm{K}_{2}$ peak and the dark absorption features which are superimposed on the bright network intersections. These dark features correspond to the dark elongated fine mottles visible in high resolution $\mathrm{H} \alpha$ filtergrams as the one shown in Figure 8. These dark fine mottles, thought by many to be identical to the spicules observed at the solar limb, the Calcium $\mathrm{K}_{2 \mathrm{v}}$ grains and other details visible on chromospheric images are all components of an incredibly complex chromosphere.

The chromosphere is a manifestation of the spectacularly rapid transition zone from the relatively cool photosphere to the hot corona and solar wind. Associated with the complexity of the chromosphere is probably a similar spatial complexity in this transition zone and in order fully to understand the origin of the solar corona, it will be necessary to resolve these spatial complexities. Our best bet for determining the physical conditions of the photosphere-chromosphere-corona transition zone lies in the observations in the ultraviolet and radio region of the spectrum. Line and continuum intensities there give very direct information on the temperature and density 


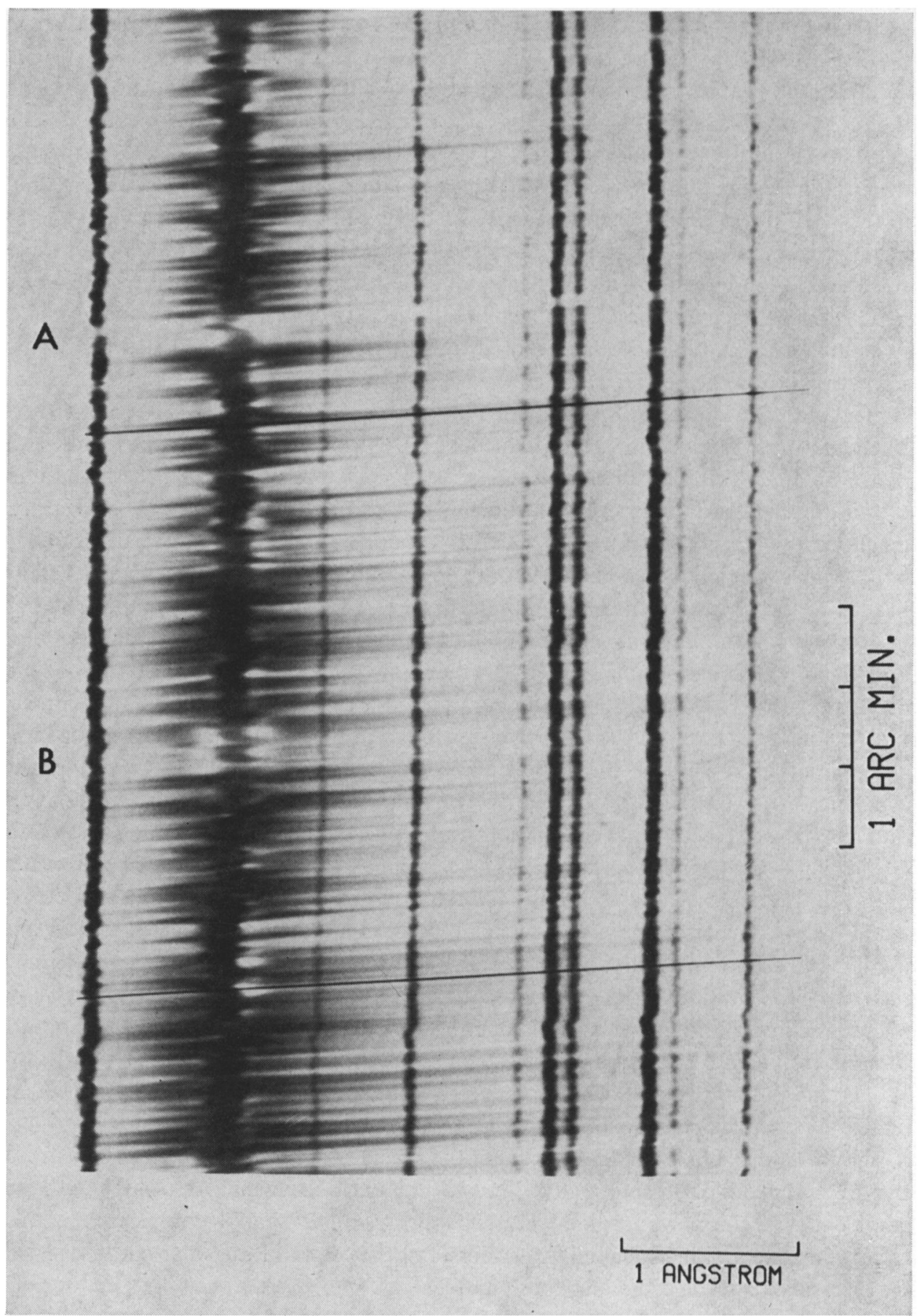

Fig. 7. Spectrum of the center of the solar disk in the $\mathrm{Ca}^{+} \mathrm{H}$ line. Blue is to the right. (Courtesy: Sacramento Peak Observatory, AFCRL.) 


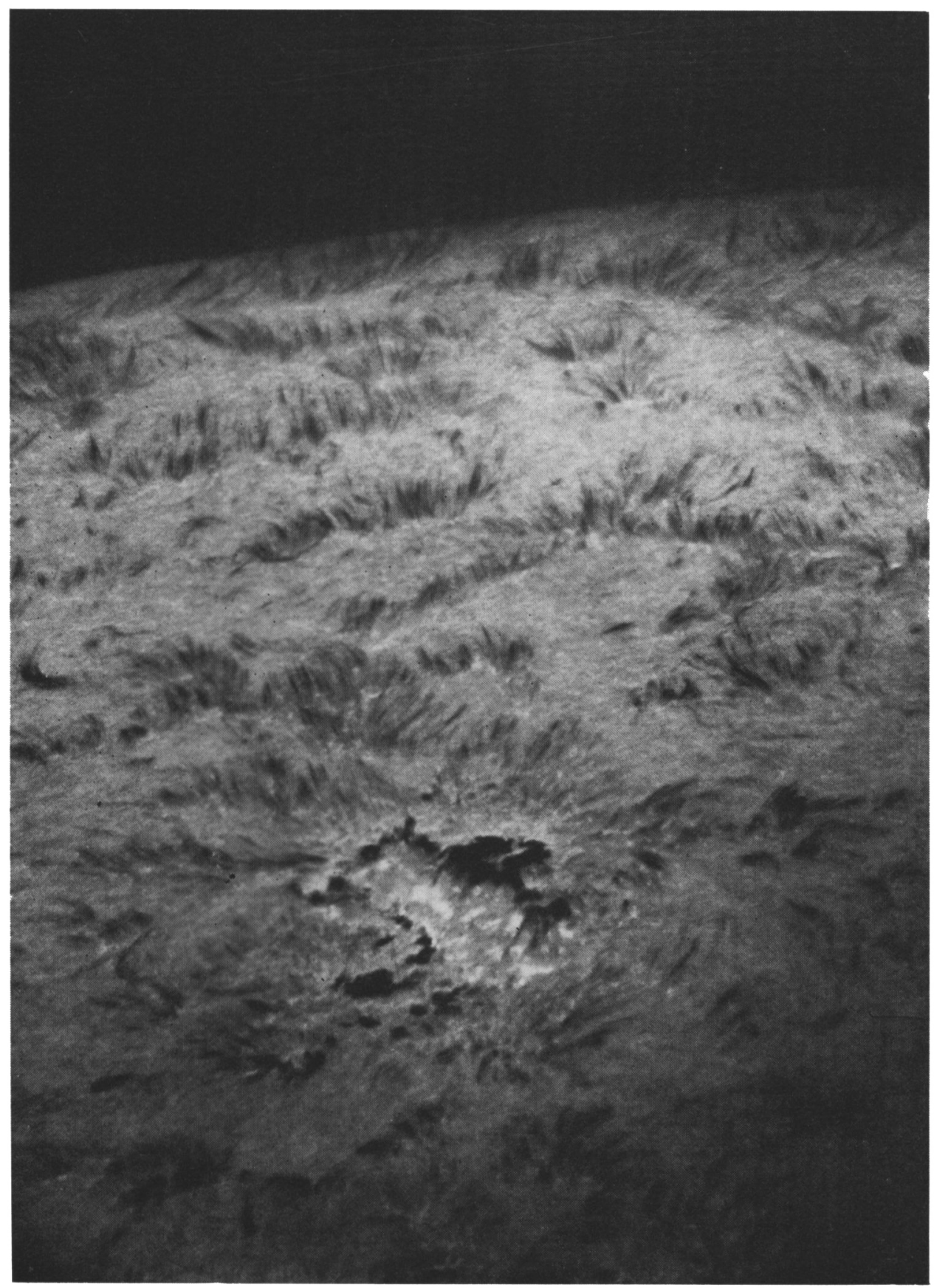

Fig. 8. Filtergram in the wing of the $\mathrm{H} \alpha$ line. (Courtesy: Sacramento Peak Observatory, AFCRL.) 


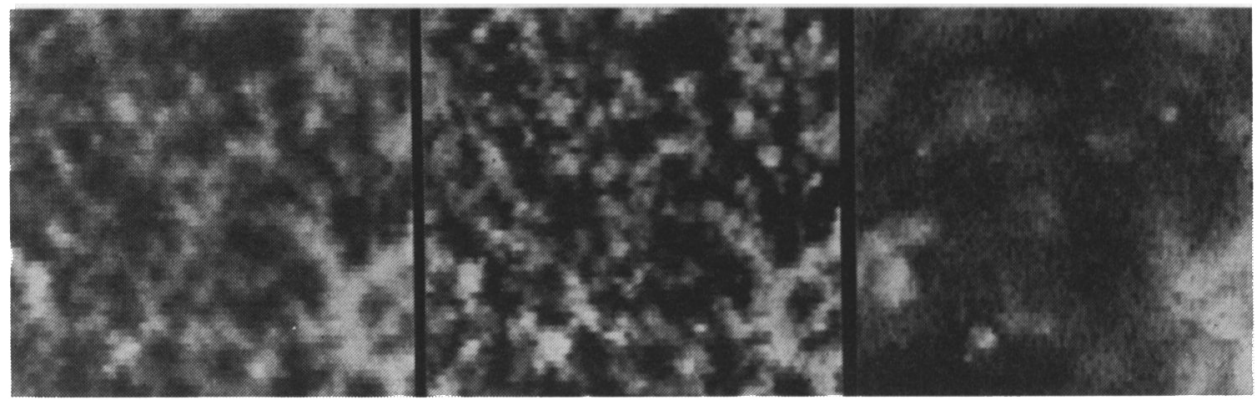

Fig. 9. Observations of the $\mathrm{L} \alpha, \mathrm{C}$ III and $\mathrm{Mg} \mathrm{x}$ chromospheric coronal network with the Harvard ATM experiment. (Courtesy: Harvard College Observatory.)

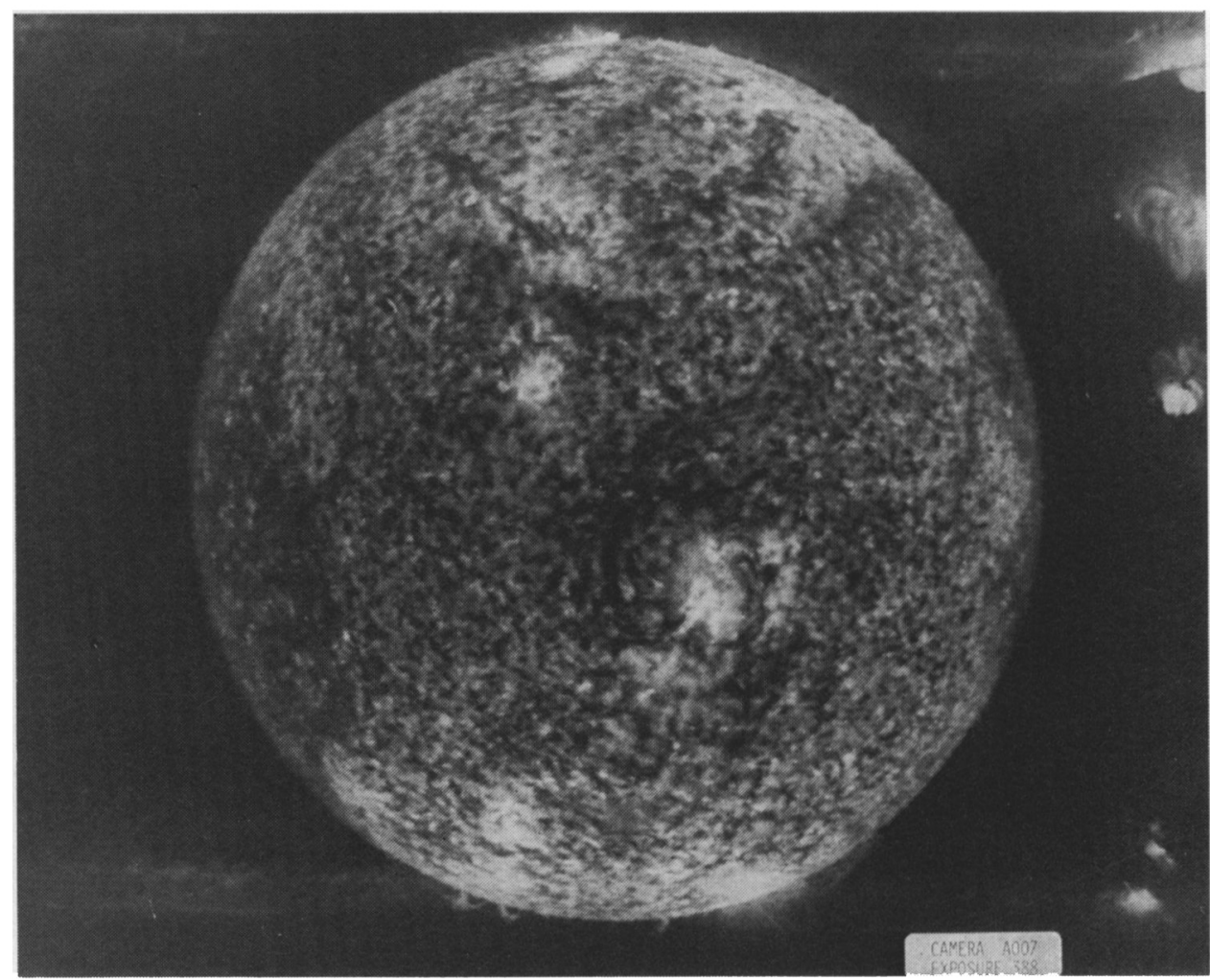

Fig. 10. Observation of the chromospheric network in He II $304 \AA$ with the NRL-ATM experiment. (Courtesy: Naval Research Laboratory.)

structure of the transition region. Recent efforts in UV and radio solar astronomy have, therefore, been in the direction of improving spatial resolution on the Sun. Especially many of the recent ATM-experiments and the experiments on the OSO-I spacecraft to be launched next year are directed toward the study of the fine structure of the transition zone. The ATM experiments have a resolution of about 5", sufficient 
to resolve the chromospheric network, and OSO-I will have a resolution of about 1 " which will be sufficient to resolve spatial details like, for example, chromospheric spicules.

Figure 9 shows examples of quiet chromosphere/corona observations with the Harvard ATM telescope. These spectroheliograms were taken simultaneously in the $\mathrm{L} \alpha$ line, a C III line and a Mg x line. The L $\alpha$ and C III lines clearly show the chromospheric network. When going to lines of ions of higher stages of ionization the network gradually fades out, its elements becoming both weaker and perhaps more diffuse until hardly any network remains in the coronal lines like the $625 \AA \mathrm{Mg} \mathrm{X}$.

Figure 10 was taken with the NRL telescope on-board ATM. This telescope gives solar images through a slitless spectrograph which are then photographed. Since it does not use a resolution limiting device like the Harvard $5^{\prime \prime} \times 5^{\prime \prime}$ slit it is in principle capable of better than $5^{\prime \prime}$ resolution. Again the network elements are clearly visible in this He II $304 \AA$ spectroheliogram.

The ATM experiments are providing solar research with a vast amount of information which will undoubtedly lead to a giant leap in our understanding of the Sun. During the course of this General Assembly and during the symposia that will follow it, we will probably see many data from ATM. We will have to wait for OSO-I to obtain at the same time 1 " spatial resolution and the spectral resolution necessary to study line profiles. From rocket experiments we have, however, already seen a sample of what to expect. Figure 11 shows a spectrum of the Sun in the $L \alpha$ line and in two lines of the $O_{I}$ triplet at $1304 \AA$, obtained by Dr Bruner. The spatial resolution of these spectra is about $20^{\prime \prime}$, the spectral resolution is sufficient to resolve the lines very well and to separate them from the central reversals caused by the geocorona.

Kilometer baseline interferometry and image synthesis as applied to solar radio astronomy opens the possibility of giving images in the 1 " resolution class. Dr Kundu is pursuing this route. Figure 12 is an example of an observation at $3.5 \mathrm{~cm}$ wavelength. In order to synthesize a fully resolved image it is, however, necessary to record data over a long time (hours) so that the temporal resolution presents a real problem, when observing the short-lived arc second features. Another potentially very exciting development in $\mathrm{mm}$ and $\mathrm{cm}$ radioastronomy is the study of the solar spectrum with high spectral resolution. Since solar spectral lines were predicted a number of years ago, there have been some, so far unsuccessful, efforts to study the spectrum in this wavelength region. If the $n \alpha$ transitions (Dupree, 1969) or any other atomic transition should be found in the radio spectrum one would have a potentially very powerful way of determining the chromospheric and coronal magnetic fields because of the very large Zeeman splitting for spectral lines in the radio region.

Some experiments on-board Skylab study the coronal structures, one by looking at the white light corona outside the solar limb, two by looking at the corona X-rays on the disk and just outside the limb. Such an X-ray image taken by AS\&E with ATM is shown in Figure 13. Spatial resolution is again in the 5" class. Both types of synoptic coronal observations will lead to vast improvement in our understanding especially of the coronal spatial and magnetic structure. An interesting possibility for studying 


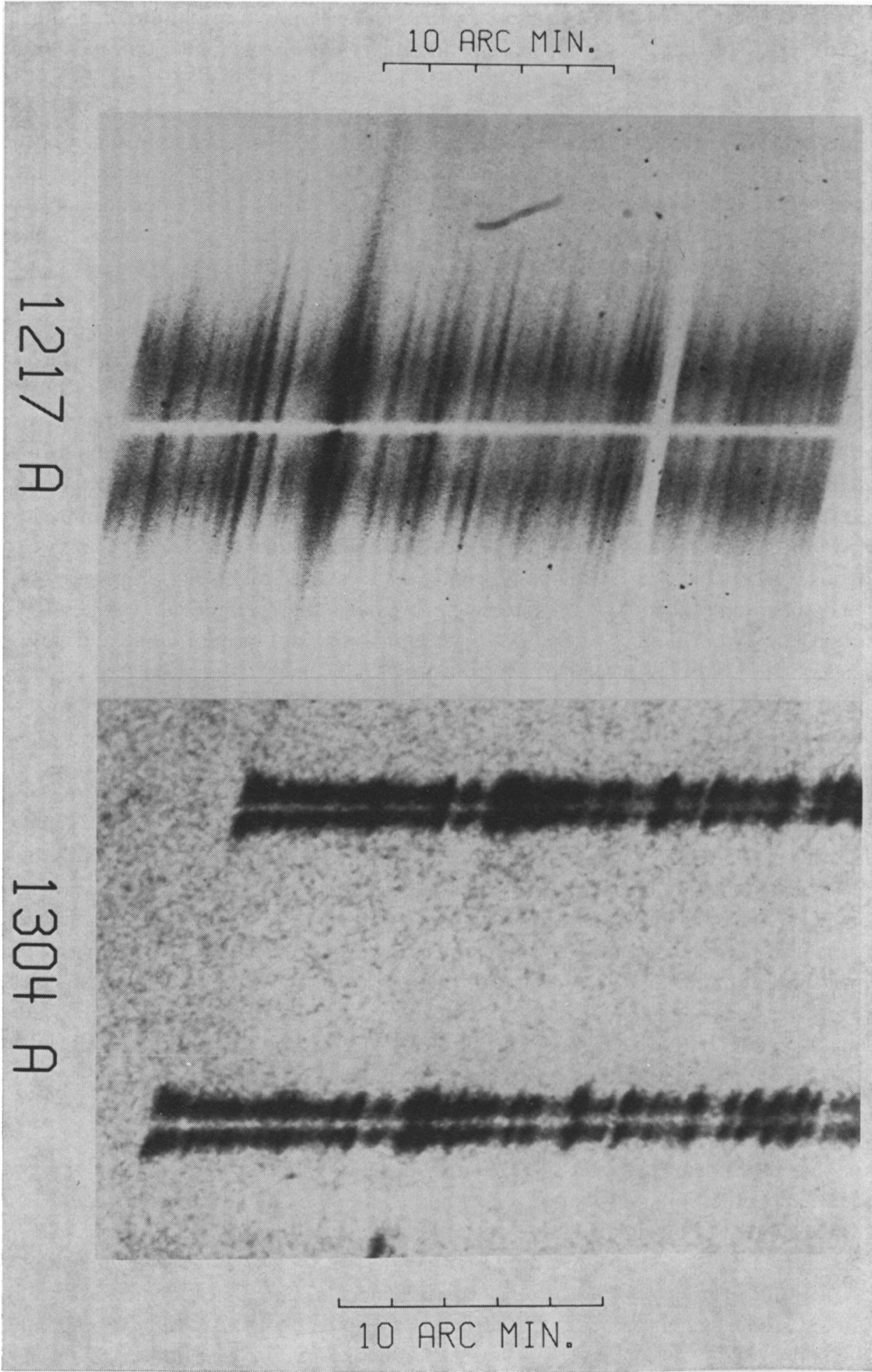

Fig. 11. Hydrogen L $\alpha$ line and two O I lines at $1304 \AA$ on the solar disk. (Courtesy: LASP, University of Colorado.) 


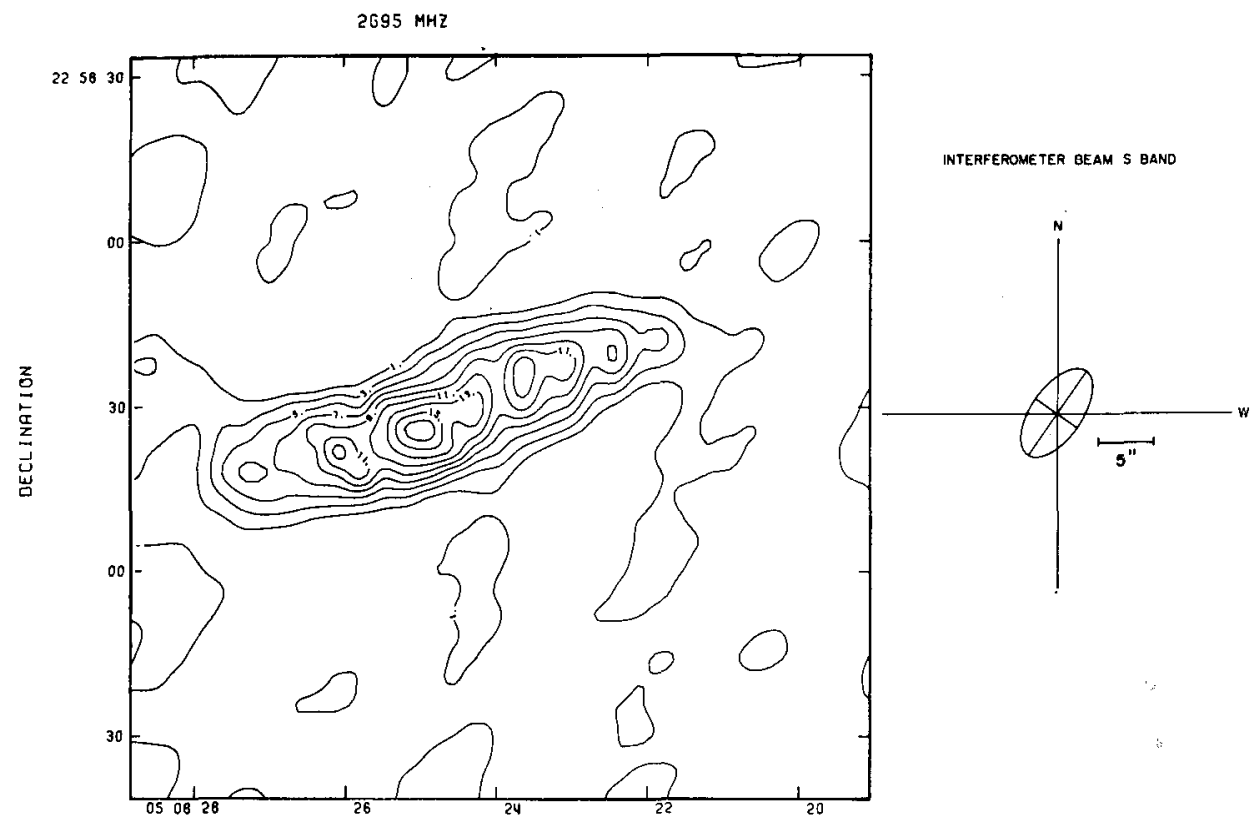

RIGHT ASCENSION

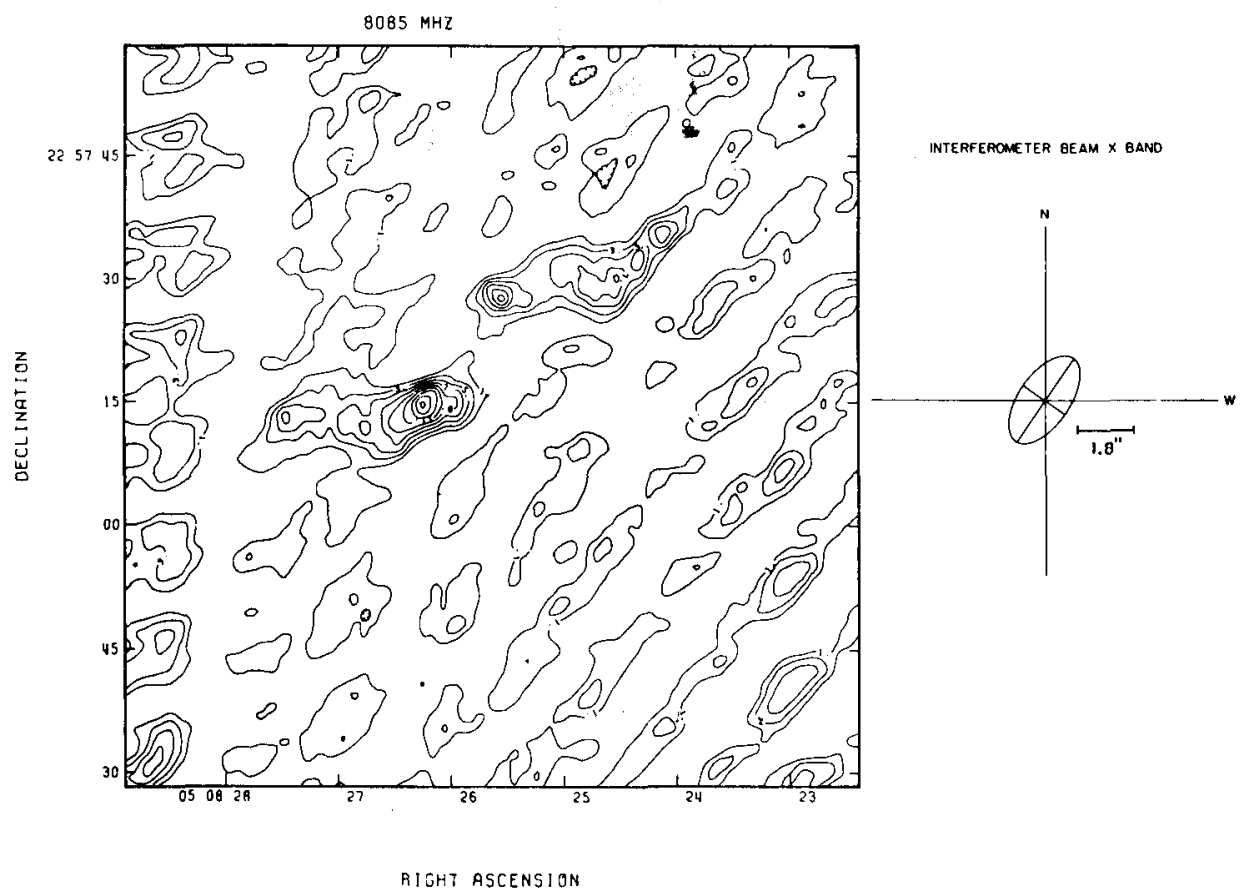

Fig. 12. Map of the solar $3.5 \mathrm{~cm}$ radiation as obtained with a long baseline interferometer. (Courtesy: University of Maryland.) 


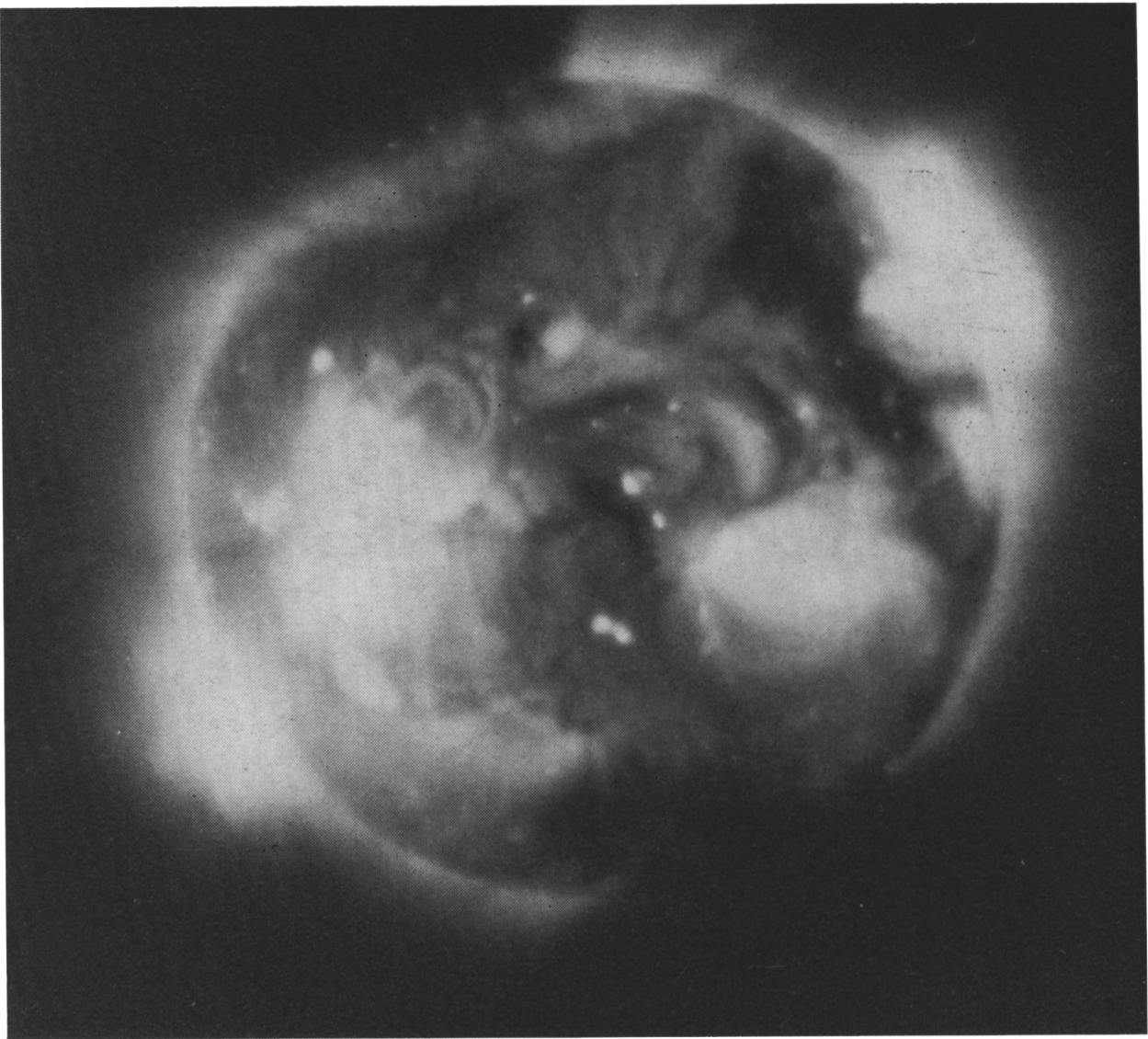

Fig. 13. X-ray image of the Sun taken from Skylab. (Courtesy: American Science and Engineering.)

the coronal temperature structure presented itself, however, during the 1970 solar eclipse when the solar $\mathrm{L} \alpha$ corona was discovered by a Culham-Harvard experiment. Figure 14 shows one of the observations. Dr Gabriel (1971) showed that the $\mathrm{L} \alpha$ emission can be explained by resonance scattering by the very few neutral hydrogen atoms left in the very hot corona. Since these atoms have very large thermal motions, the line width of the coronal $L \alpha$ line should be a very accurate indicator of the coronal temperature.

With this I terminate what necessarily had to be a rather superficial summary of the present status of solar observational research. Undoubtedly, I am guilty of the omission of some significant efforts in this area on the one hand and maybe of overstressing some of my own interests on the other hand, In summary, I do believe that the main overall stress in observational solar work has been on improving spatial resolution of solar observations with a secondary significance attached to the improvement of spectral resolution. 


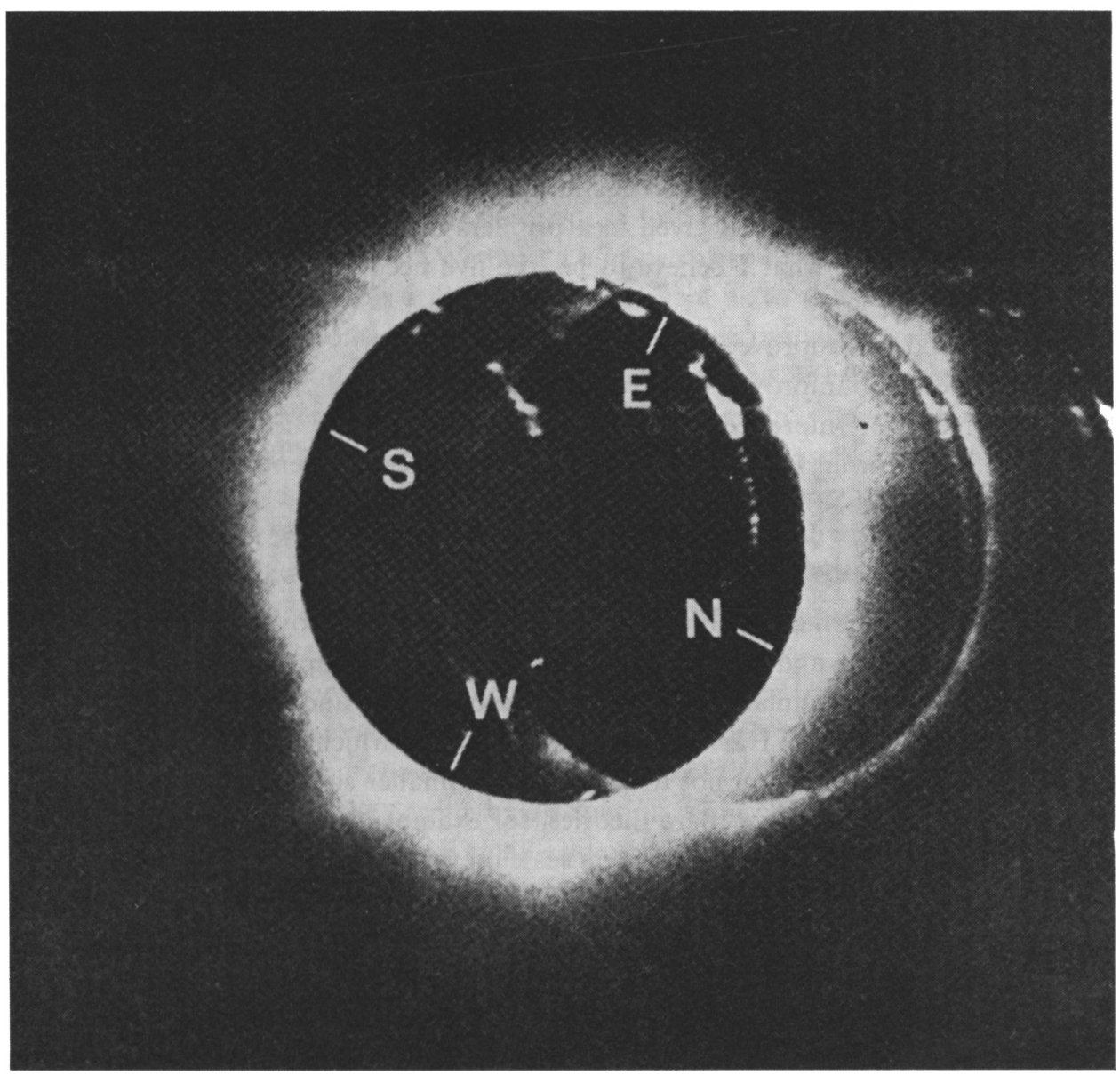

Fig. 14. L $\alpha$ image of the solar corona. (Courtesy: Culham Laboratories.)

\section{Future Objectives of Observational Solar Research}

One obvious way to prophesy on the future developments of observational solar research is to extrapolate from the past. In doing this one would predict a continued improvement, especially in the spatial but also in the spectral, resolution of solar observations. Solar physicists doing the observations tend to follow this line since it leads into one of the major unexplored areas of solar research - that of the subgranular, microstructure of the Sun - in which major discoveries are likely.

Solar physicists who concentrate on the theoretical aspect of the sun are frequently frightened by the complexities resulting from high resolution observations. Theoretical solar physics has not reached a sufficient level of sophistication to deal with these complexities. The need for higher resolution is questioned by both theorists, the sponsoring agencies, and the non-solar astrophysicists who are competing for in- 
strumentation funds. The solar observationalist is thus forced to justify his endeavors beyond the desire to explore the unknown. He is asked to describe the problems which he wants to solve and to show that these problems are relevant in some broader frame of values. Based on this, he then justifies his efforts to obtain novel and new instruments. In his request to me to give this lecture, Dr Athay, a theorist par excellence, indicated his desire to have it given in a problem-oriented framework. I will try to do this by discussing what I believe to be 'the five needs of observational solar astronomers'. These are:

(a) The Need for Improved Spatial Resolution,

(b) The Need for Improved Spectral Resolution,

(c) The Need for Improved Temporal Resolution,

(d) The Need for an Improved Interpretation of Measurements, and

(e) The Need for Novel Observations and Ideas.

\subsection{THE NEED FOR IMPROVED SPATIAL RESOLUTION}

Whenever it has become possible to study the Sun with improved spatial resolution we have seen smaller and smaller details. Most recent examples of this are the observations of the subgranular structures (discussed already) and the results of the solar speckle interferometry by Harvey et al. (1973) both of which resolved solar features near $0.2^{\prime \prime}$. There is no reason not to expect much smaller size structures of the solar atmosphere. On the contrary, flare theories, for example, predict that current sheets associated with the flare instability have a scale on the order of $1 \mathrm{~km}$ and less. We can also infer from spectral line width observations that small scale structures must exist, since the power in the spatial velocity spectrum for the highest resolution observations falls well short of the total power for all spatial frequencies as derived from the line broadening (see e.g. Beckers et al., 1969).

Very small structures, therefore, undoubtedly exist on the Sun. It may, however, be impossible to observe them since the radiation used to observe the Sun represents an integration over a long effective path along the line of sight. The contribution function half-width is typically about 2 scale heights corresponding to $150 \mathrm{~km}$ or $0.2^{\prime \prime}$ in the photosphere. When looking at a 3 dimensionally and isotropically random structure like turbulence it would, therefore, become difficult to resolve anything below this size. This is, however, not necessarily true for all small-scale structures like thread type structures in flares and prominences. Fine structures seen in the corona, in fact, already give an example of observed phenomena which are much smaller than the contribution function halfwidth.

Given the fact that small structures are present and observable, why should an effort be made to study them? I see three broad areas of interest which would require higher resolution observations. The first is the need to know the three-dimensional model of the solar atmosphere. Only through the knowledge of the local variations in the atmospheric physical conditions like temperature and density will we be able to properly assess the validity for both stellar and solar physics of the inferences drawn on the basis of simple one-dimensional atmospheric models. 
The second reason for improving our spatial resolution lies in the need to fill in major gaps in our understanding of the energy transport in the outer layers of the Sun and stars. Radiation carries the bulk of the energy away from the Sun. The motion in the photosphere, resulting in the relatively small kinetic energy flow, is, however, responsible for the existence of the solar chromosphere, corona, and solar wind and associated phenomena like angular momentum loss. What are the characteristics of the motions in the photosphere? We have a fair idea as to the properties of the supergranulation although there is still some disagreement as to the nature of these flow patterns. It has been customary in the last decade to think of the photospheric granulation as a direct display of the convection elements of the solar hydrogen convection zone. Recently, however, the suggestion that the granulation is the result of waves has been getting renewed attention. It seems to me that new observations of exploding granules and of rapid horizontal granular motions lend support to the notion of the granulation as the results of waves which in turn are excited by the subphotospheric turbulence or convection. High resolution observations are needed to decide between these two granule models. The nature of the subgranular structures is entirely a matter of speculation.

Which size elements are responsible for the heating of the transition region and the corona? As far as I am aware, all coronal heating models are one-dimensional. The effect on coronal heating of the horizontal scale size of the waves that excite the shock waves which in turn heat the corona by dissipation is not clear to me. It could well be that the interaction and collision of small-scale shocks would enhance the effectiveness of this heating mechanism, in which case the granular and subgranular elements, which contain much of the kinetic energy flow, are dominant. This is an area of speculation on which I invite comments. There are, however, also observational indications that the heating occurs at a small scale. Both Liu (1973) and Cram (1973) have suggested that the small grains visible in the violet wing of the $\mathrm{H}$ and $\mathrm{K}$ lines are the results of shocks.

Knowledge of the temperature and density structure of the transition zone is of great value when evaluating the corona heating processes. It permits us to study the rate of energy dissipation and of energy transport by conduction and perhaps kinetic processes. Observations of the emission measures of lines of widely varying ionization and excitation potential in the ultraviolet have given us an excellent tool for the study of this temperature and density structure. So far, most studies have been one-dimensional, although it is clear from satellite and rocket observations that there are very large horizontal fluctuations in the emission measure. Hence there are probably variations in the energy transport down to the scale of spicules and calcium grains and perhaps smaller structures. The emission measure technique of probing the transition zone is directly applicable to any size structure so that high resolution observations in the ultraviolet will give direct information on the small scale structure of the transition zone.

The third reason for improving the spatial resolution comes from the need for understanding the mass transport in the Sun. The loss of mass in solar and stellar winds is 
directly related to the presence of non-radiative energy transport. Mass and energy transport are therefore related. Solar spicules carry two orders of magnitude more matter into the corona than is lost by the solar wind. They are, therefore, very significant in the mass balance. We know nothing about the mass transfer by other photospheric, chromospheric and coronal structures. Nor is the relation between mass and energy input in the corona clear. Do they occur in the same region or do they occur spatially quite independently?

In order to increase our understanding of the structure of the solar atmosphere and of the energy and mass transfer in solar and stellar atmospheres, it is necessary to improve our spatial resolution. Large aperture optical telescopes are needed for photospheric studies. The introduction of ground-based vacuum telescopes has been very successful and it is likely that proper site selection will make it possible fully to exploit vacuum telescopes with apertures larger than 1 meter. Good time sequences will probably require more expensive balloon and satellite-borne telescopes. The study of the transition zone requires high resolution satellite-borne ultraviolet telescopes. ATM and OSO-I will produce 1 " class observations. The Greenstein Report recommended higher resolution OSO-type spacecraft, especially to study the transition zone and solar flares. It seems also that a continued effort for better resolution in solar radio astronomy might supply very significant additional information on the solar chromosphere.

\subsection{THE NEED FOR IMPROVED SPECTRAL RESOLUTION}

In contrast to the spatial resolution it is possible to give definite limits to the desired spectral resolution. Thermal broadening limits the required spectral resolution to $\approx 10^{5}$ in the photosphere and $\approx 2 \times 10^{3}$ in the corona unless extremely precise profiles are needed in which case an order of magnitude larger resolution is desirable. OSO-I will give spectral resolutions approaching these values down to about $1000 \AA$. From the OSO-I investigation one hopes to obtain additional information on velocity patterns in the transition zone which in turn will lead to information regarding mass transport and wave dissipation. In the optical region we have now all the spectral resolution which one could possibly desire. The areas of future developments seem to be (a) continued improvements of the spectral resolution especially in the far ultraviolet and X-ray spectrum, especially when combined with high spatial resolution, and (b) the analysis of the microwave and mm radio spectrum, especially as concerned with the search of solar absorption and emission lines there.

\subsection{THE NEED FOR IMPROVED TEMPORAL RESOLUTION}

Smaller and smaller structures have shorter and shorter lifetimes. The temporal resolution of solar telescopes is generally, however, sufficient to resolve the temporal variations occurring on the Sun. Flux limitations sometimes present a problem which requires larger telescope apertures for a solution. Frequently image variations caused by non-solar origin like seeing and spacecraft motions also require high time resolution. 


\subsection{THE NEED FOR IMPROVED INTERPRETATION OF THE MEASUREMENTS}

Solar observations consist in the first instance of intensity measurements as a function of position on the Sun, time, wavelength and polarization mode. To infer from these the physical conditions on the Sun is often difficult even in such commonly considered simple cases as Doppler shift and Zeeman splitting observations. One real need for the observational solar physicist is a better insight into the meaning of his measurements so that he may avoid the various traps associated with simple-minded interpretation. Let me illustrate this need in the case of Doppler shift and Zeeman splitting measurements.

All measurements are out of necessity a spatial average over an area of the Sun. All indications are that any area which can presently be resolved must contain velocity (or magnetic field) inhomogeneities so that the measurement gives a velocity (or magnetic field) which represents a spatial average, weighted by factors which include local variations in line strength and continuum intensity. One may not, therefore, conclude from the existence of an average blue shift that this corresponds to an average mass flux in the direction of the observer. The most spectacular example of this trap is the blue shift of the Sun itself which is believed to be caused by the upward motions of the bright granules dominating, as far as the average spectral intensities are concerned, the downward motion of the intergranular regions. In weak lines this blue shift amounts to $0.5 \mathrm{~km} \mathrm{~s}^{-1}$. If interpreted as an expansion of the Sun this would mean that the sun would expand out to $1 \mathrm{AU}$ in about 10 years. Since the blue shift observations were made over 17 years ago we can be sure that this interpretation is wrong. Other examples of trouble caused by spatial averaging are the inconsistencies in the magnetic fluxes observed in different lines (Harvey et al., 1969) and the measurements of the Evershed Effect. The only real solution to the problem of spatial averaging is to avoid the averaging by improving the spatial resolution. This is another good reason for improving the spatial resolution. Since it is unlikely that one will ever resolve the Sun fully we will also have to continue the past approach which makes sophisticated guesses as to the spatial structure based on the observations in different lines.

Temporal averaging presents, in principle, a similar trap. If velocity variations with time are so fast that we cannot resolve them, one can have the same trouble as with spatial averaging. One example of this effect is perhaps the Evershed Effect which Maltby et al. (1967) explained as a temporally and spatially unresolved wave phenomenon.

There are other difficulties in the interpretation of data even when the temporal and spatial resolution is sufficient. First it should be remembered that downward motions, observed in the $\mathbf{H} \alpha$ lines at the supergranule boundaries, only refer to the motions of the neutral hydrogen atoms responsible for the $\mathrm{H} \alpha$ line and not to an overall mass flux as has often been suggested. The downward $\mathrm{H} \alpha$ motions may in fact be compensated by upward motions in gases which are invisible in the $\mathrm{H} \alpha$ line. Then there is averaging over height which causes real problems in case of a variation of the Doppler 
velocity along the line of sight. Frequently contribution function arguments are being used to estimate the effective optical depth to which a Doppler shift measurement in a particular line refers. It can be shown (Parnell et al., 1969) that the effective optical depth is a function of the velocity variation along the line of sight and that it very well can fall outside the main part of the contribution function. In addition, there are cases in which the apparent measured velocity does not correspond to an actual velocity at any point along the line of sight. Athay (1970) and myself (Beckers, 1968) showed that this may frequently be the case in the $\mathrm{H} \alpha$ line where even the direction of an apparent motion or magnetic field can be opposite to the real motion or magnetic field.

Apart from improving the spatial and temporal resolution, the best progress in this area can be made in line profile calculations of two- and three-dimensional solar model atmospheres in which various velocity and magnetic field distributions are assumed. Hopefully, one may gain some insight as to the meaning of the measurements by comparing the profiles and shifts of carefully selected lines with these calculations.

\subsection{THE NEED FOR NOVEL OBSERVATIONS AND IDEAS}

The final need for observational solar research lies in the need for new types of observations and for new ideas. So far I have just extrapolated from past experiences into the future. The most exciting progress is often made, however, with entirely new experiments and ideas. Examples are the solar neutrino experiment and the attempts to detect solar oblateness. Less spectacular examples of new experiments are perhaps the various developments of Stokes polarimeters and an attempt to study the intensity and profile of the coronal $L \alpha$ line at the 1974 solar eclipse. New ideas and experiments can be the result of a close interaction with other fields of scientific endeavor including theoretical astrophysics, fluid dynamics, optics, etc.

\section{Conclusion}

I have refrained in this review from discussing details of potential future solar instruments. Instead, I indicated what, in my opinion, the general direction should be in which solar observational research should go. There are already a number of efforts underway which are consistent with my suggestions. It takes a lead time on the order of a decade to obtain most instruments of the magnitude suggested in this review. It is in a way, therefore, a futile effort to discuss the aims for the next decade since most of the plans for the next decade are already firm.

Most new instrumental efforts are expensive. As Greenstein repeatedly stated in his study 'Astronomy is Big Science'. In recommending a program for science, the big items are commonly stressed since these require the main promotion effort. Much can, however, be done with more modest means - especially in connection with the last two needs of observational solar astronomy, the need for novel observations and ideas, and the need for an improved understanding of the observations. 


\section{References}

Athay, R. G.: 1970, Solar Phys. 12, 175.

Beckers, J. M.: 1968, Solar Phys. 3, 367.

Beckers, J. M.: 1973a, Bull. Am. Astron. Soc. 5, 269.

Beckers, J. M.: 1973b, J. Opt. Soc. Am. 63, 484.

Beckers, J. M. and Parnell, R. L.: 1969, Solar Phys. 9, 39.

Coates. R. J., Gibson, J. E., and Hagen, J. P.: 1958, Astrophys. J. 128, 406.

Cram, L.: 1973, private communication.

Dupree, A.: 1969, Astrophys. J. 152, L125.

Gabriel, A.: 1971, Solar Phys. 21, 392.

Gabriel, A. H. et al.: 1971, Astrophys. J. 169, 595.

Harvey, J. W. and Breckenridge, J. B.: 1973, Astrophys. J. 182, L 137.

Harvey, J. W. and Livingston, W.: 1969, Solar Phys. 10, 283.

Kundu, M.: 1973, in R. G. Athay (ed.), 'Chromospheric Fine Structure', IAU Symp. 56, in press. Liu, Su Yang: 1973, Astrophys. J., in press.

Maltby, P. and Erickson, G.: 1967, Solar Phys. 2, 249.

Noyes, R. W., Beckers, J. M., and Low, F. J.: 1968, Solar Phys, 3, 36.

Parnell, R. L. and Beckers, J. M.: 1969, Solar Phys. 9, 35.

Simon, G. W. and Zirker, J. B.: 1973, Solar Phys., in press. 